\title{
The Cauchy problem for weakly hyperbolic systems
}

\author{
F.Colombini, Guy Métivier ${ }^{\dagger}$
}

October 14, 2016

\begin{abstract}
We consider the well-posedness of the Cauchy problem in Gevrey spaces for $N \times N$ first order weakly hyperbolic systems. The question is to know wether the general results of M.D.Bronštein [Br] and K.Kajitani [Ka2] can be improved when the coefficients depend only on time and are smooth, as it has been done for the scalar wave equation in [CJS]. The anwser is no for general systems, and yes when the system is uniformly diagonalizable: in this case we show that the Cauchy problem is well posed in all Gevrey classes $G^{s}$ when the coefficients are $C^{\infty}$. Moreover, for $2 \times 2$ systems and some other special cases, we prove that the Cauchy problem is well posed in $G^{s}$ for $s<1+k$ when the coefficients are $C^{k}$, which is sharp following the counterexamples of S.Tarama [Ta1]. The main new ingredient is the construction, for all hyperbolic matrix A, of a family of approximate symmetrizers, $S_{\varepsilon}$, the coefficients of which are polynomials of $\varepsilon$ and the coefficients of $A$ and $A^{*}$.
\end{abstract}

MSC Classification : 35 L 50, 35 L 45, 35 L 40.

Keywords: hyperbolic systems, Cauchy problem, symmetrizers, well posedness, Gevrey spaces.

\section{Introduction}

It is now well known that the Cauchy problem is not necessarily well posed in $C^{\infty}$ for general weakly hyperbolic equations or systems (see e.g. [CDGS]

${ }^{*}$ Università di Pisa, Dipartimento di Matematica, Largo B.Pontecorvo 5, 56127 Pisa, Italy, ferruccio.colombini@unipi.it .

†Université de Bordeaux - CNRS, Institut de Mathématiques de Bordeaux, 351 Cours de la Libération , 33405 Talence Cedex, France, guy.metivier@math.ubordeaux.fr.

${ }^{\ddagger}$ The second author thanks il Dipartimento di Matematica della Università di Pisa for his hospitality 
[CS] for counterexamples and [IvPe] for necessary conditions). On the other hand, in [Br], M.D.Bronštein has proved that the Cauchy problem for weakly hyperbolic equations and systems is well posed in Gevrey spaces $G^{s}$ for $s \leq 1+1 /(m-1)$ where $m$ is the maximum multiplicity of the characteristics, provided that the coefficients themselves have the Gevrey regularity $G^{s}$ with respect to the space variables and are sufficiently differentiable in time, see also [Ka1] for systems. A different proof, based on symmetrizer technics, is given in $[\mathrm{CNR}]$. The result has also been extended to nonlinear systems, [Ka3].

For systems, not only the algebraic multiplicities are important, but also the diagonalizability properties play a role. In particular, for uniformly diagonalizable systems, it is shown in [Ka2] that the Cauchy problem is well posed in $G^{s}$, for $s<2$, independently of the multiplicities.

It has been noticed that the general result can be improved in some special cases, in particular when the coefficients depend only on time. For the wave equation

$$
\partial_{t}^{2} u-\sum a_{j, l}(t) \partial_{x_{j}} \partial_{x_{l}} u=f,
$$

with $\sum a_{j, l}(t) \xi_{j} \xi_{l} \geq 0$ for all $t$ and $\xi$, it is proved in [CJS] that the Cauchy problem is well posed in Gevrey spaces $G^{s}$ for $s<1+k / 2$ if the coefficients $a_{j, k}$ are $C^{k}$. In the same paper, the authors prove that the threshold index $1+k / 2$ is sharp. This result has been extended to $2 \times 2$ uniformly diagonalizable systems in [CoNi]. However, S.Tarama has proved in [Ta1] that the Cauchy problem is well posed in Gevrey classes $G^{s}$ for $s<1+k$ for a class of $2 \times 2$ uniformly diagonalizable systems, and, by counterexamples, that this index is sharp. We will show that Tarama's result extends to all $2 \times 2$ uniformly diagonalizable weakly hyperbolic systems.

More generally, this paper is concerned with the well posedness of the Cauchy problem in Gevrey spaces for $N \times N$ weakly hyperbolic systems

$$
L u:=\partial_{t} u+\sum_{j=1}^{d} A_{j}(t) \partial_{x_{j}} u=\partial_{t} u+A\left(t, \partial_{x}\right) u .
$$

The matrices $A_{j}$ are defined for $t$ in some interval $[0, T]$. Below, we always assume that $L$ is weakly hyperbolic, that is that for all $t$ and $\xi \in \mathbb{R}^{d}$, the eigenvalues of $A(t, \xi)=\sum \xi_{j} A_{j}(t)$ are real.

Before stating our results, let us recall some definitions. For $s>1$ and $\Omega \subset \mathbb{R}^{d}$, the Gevrey space $G^{s}(\Omega)$ is the set of $C^{\infty}$ functions $u$ on $\Omega$ such that for all compact set $K \subset \Omega$ there is a constant $C$ such that

$$
\forall \alpha \in \mathbb{N}^{d}, \quad\left\|\partial_{x}^{\alpha} u\right\|_{L^{\infty}(K)} \leq C^{|\alpha|+1}(|\alpha| !)^{s} .
$$


We denote by $G_{0}^{s}(\Omega)$ the subset of functions which are compactly supported in $\Omega$. For functions depending also on time, $u(t, x)$ with $(t, x) \in \tilde{\Omega}$ a relatively open subset of $\left[0, T\left[\times \mathbb{R}^{d}\right.\right.$, we say that $u \in C^{0} G^{s}(\tilde{\Omega})$ if all the derivatives $\partial_{x}^{\alpha} u$ are continuous on $\tilde{\Omega}$ and for all compact set $\tilde{K} \subset \tilde{\Omega}$ there is a constant $C$ such that

$$
\forall \alpha \in \mathbb{N}^{d}, \quad\left\|\partial_{x}^{\alpha} u\right\|_{L^{\infty}(\tilde{K})} \leq C^{|\alpha|+1}(|\alpha| !)^{s} .
$$

Note that it is required that these estimates are valid up to $t=0$ (when there are such points in $\tilde{\Omega}$ ).

We use the following terminology:

Definition 1.1. We say that the Cauchy problem for (1.2) is locally wellposed in $G^{s}$ if for all neighborhood $\Omega \subset \mathbb{R}^{d}$ of the origin, there is a neighborhood $\tilde{\Omega}$ of 0 in $\left[0, T\left[\times \mathbb{R}^{d}\right.\right.$ such that for all $\left.f \in C^{0} G^{s}(\tilde{\Omega})\right)$ and $h \in G^{s}(\Omega)$ the problem

$$
L u=f, \quad u_{\mid t=0}=h,
$$

has a unique solution $u \in C^{0} G^{s}(\tilde{\Omega})$.

We say that the problem is globally well posed in $G^{s}$ if for $\Omega=\mathbb{R}^{d}$ one can take $\tilde{\Omega}=\left[0, T\left[\times \mathbb{R}^{d}\right.\right.$.

Our main question is to know wether the smoothness in time of the coefficients improves Bronštein's threshold index $m /(m-1)$. In general the answer is no:

Theorem 1.2. There are weakly hyperbolic systems (1.2) with analytic coefficients such that the Cauchy Problem is not locally well posed in $G^{s}$ for $s>N /(N-1)$.

This result is quite elementary, but not in the literature to the knowledge of the authors. It is proved in Section 2. The idea is that, in general, the variation in time of the coefficients have the same effect as adding a general zero-th order term $B$ to $L$, and even in the constant coefficient case, the well posedness is stable by such perturbations only if $s \leq m /(m-1)$.

This shows the importance of the uniform diagonalizability assumptions in the papers [Ka2, CoNi, Ta1] cited above, because in sharp contrast, the well-posedness of the Cauchy problem remains valid for all bounded perturbation $B(t)$. Recall the definition:

Definition 1.3. The system (1.2) is said to be uniformly diagonalizable if for all $t$ and $\xi$ the matrix $A(t, \xi)$ is diagonalizable and the eigenprojectors are bounded uniformly with respect to $t$ and $\xi$. 
There are several equivalent conditions (see e.g. [Me] where this condition is called strong hyperbolicity of the symbol). One of them is that there exists a bounded family of symmetrizers, that is a family of self adjoint and positive matrices $S(t, \xi)$ such that $S(t, \xi) A(t, \xi)$ is self adjoint and $S$ and $S^{-1}$ are uniformly bounded.

Under this condition, the Gevrey index can be improved when the coefficients are smooth in time. For $k \in \mathbb{N}, \mu \in] 0,1]$, we denote by $C^{k, \mu}([0, T])$ the space of $C^{k}$ functions on $[0, T]$ such that their $k$-th derivative satisfies a Hölder condition of order $\mu$ (Lipschitz if $\mu=1$ ).

When $k+\mu>m(m-1)$, the following result improves the general result of K.Kajitani [Ka2] who proved the well posedness for $s<2$.

Theorem 1.4. Consider a uniformly diagonalizable weakly hyperbolic system (1.2), with $C^{k, \mu}$ coefficients. Then, for all bounded matrix $B(t)$, the Cauchy problem for $L+B$ is locally and globally well posed in Gevrey spaces $G^{s}$ with $s<1+(k+\mu) / m(m-1)$ where $m$ is the maximal multiplicity of the eigenvalues.

Corollary 1.5. Consider a uniformly diagonalizable weakly hyperbolic system (1.2), with $C^{\infty}$ coefficients. Then, for all bounded matrix $B(t)$, the Cauchy problem for $L+B$ is locally and globally well posed in all Gevrey spaces $G^{s}$ with $s \in[1, \infty[$.

When $m=2$ the computations are more explicit and we are able to get a better control of the symmetrizers. We will obtain in Section 5 the well posedness for $s<1+k+\mu$ for coefficients in the class $C^{k, \mu}$. This is sharp by S.Tarama's result [Ta1].

Theorem 1.6. Consider a uniformly diagonalizable weakly hyperbolic system (1.2) with coefficients in $\left.\left.C^{k, \mu}([0, T]), k \in \mathbb{N}, \mu \in\right] 0,1\right]$. If the multiplicity of the eigenvalues is at most 2 , then, for all bounded matrix $B(t)$, the Cauchy problem is locally and globally well posed in Gevrey spaces $G^{s}$ with $s<1+k+\mu$.

In particular, this extends Tarama's result [Ta1] to general $2 \times 2$ weakly hyperbolic systems. We will also show that the threshold index $1+k+\mu$ is also valid for a special class of $N \times N$ systems (see Theorem 7.4 below). For general $m$, the index $1+(k+\mu) / m(m-1)$ is likely to be not optimal, but it is sufficient to imply Corollary 1.5.

The paper is organized as follows. We prove Theorem 1.2 in Section 2. Next, in Section 3, we reduce the proof of the well-posedness in $G^{s}$ to the 
construction of approximate symmetrizers $S(t, \xi)$ for the matrices $A(t, \xi)=$ $\sum \xi_{j} A_{j}$. In practice, for $|\omega|=1$, one constructs families of approximate symmetrizers $S_{\varepsilon}(t, \omega)$ of $A(t, \omega)$ depending on the parameter $\varepsilon>0$ and next one chooses

$$
S(t, \xi)=S_{\varepsilon}(t, \omega), \quad \omega=\frac{\xi}{|\xi|}, \quad \varepsilon=|\xi|^{-\gamma} .
$$

Two conditions are in competitions in the proof of the energy estimate:

$$
\left\{\begin{array}{l}
\operatorname{Im} S_{\varepsilon}(t, \omega) A(t, \xi) \lesssim \varepsilon|\xi| S_{\varepsilon}(t, \omega), \\
\partial_{t} S_{\varepsilon}(t, \omega) \lesssim \varphi_{\varepsilon}(t, \omega) \varepsilon^{-\beta} S_{\varepsilon}(t, \omega)
\end{array}\right.
$$

where the $\varphi_{\varepsilon}(\cdot, \omega)$ are bounded in $L^{1}$. The exponent of $\varepsilon$ in the first estimate is just a normalization, while the exponent $\beta$ is the key element of the analysis. One chooses $\gamma$ in (1.6) to balance the two terms $\varepsilon|\xi|$ and $\varepsilon^{-\beta}$, that is $\gamma=1 /(\beta+1)$, so they are both equal to $|\xi|^{\alpha}$ with $\alpha=\beta /(\beta+1)$. By Gronwall's lemma, the amplification factor for the o.d.e. deduced by Fourier transform of equation $L u=0$, is $e^{C|\xi|^{\alpha}}$. From here, one deduces the existence of solutions when the Fourier transforms of the data decay faster than $e^{-C|\xi|^{\alpha}}$, and finally when the data belong to $G^{s}$ when $s<1 / \alpha=1+1 / \beta$.

For Lipschitz coefficients and uniformly diagonalizable systems, one easily construct symmetrizers such that $\partial_{t} S_{\varepsilon} \lesssim \varepsilon^{-1} S_{\varepsilon}$, recovering the well posedness for $s<2$ obtained in general in [Ka2]. All our analysis aims to improve this estimate of the time derivative of the symmetrizers. For this, the new ingredient is the construction of new families of symmetrizers, the coefficients of which are polynomials of $\varepsilon$ and of the coefficients of $A(t, \omega)$, thus of class $C^{k, \mu}$. Because of this regularity, and using the positivity of $S_{\varepsilon}$, we can use the estimates of [CJS, Ta2], to obtain the bound in (1.7) for $\partial_{t} S_{\varepsilon}$ with a parameter $\beta<1$, at least if $k$ is large enough. The Colombini-Jannelli-Spagnolo-Tarama estimate is recalled in Section 4, while the construction of symmetrizers is performed in Sections 5 to 7, first for $2 \times 2$ systems, and then in general.

\section{The general case}

For general $N \times N$ system and maximal multiplicity $N$, the threshold index is $N /(N-1)$ the index given by Bronštein's theorem.

Proof of Theorem 1.2. The counterexample is in dimension $d=1$. Consider 
the nilpotent $N \times N$ matrix

$$
A_{1}=\left(\begin{array}{cccc}
0 & 1 & 0 & \\
& 0 & \ddots & \\
& & \ddots & 1 \\
& & & 0
\end{array}\right)
$$

and the rotations in the plane generated by the first and the last vector of the basis:

$$
\Omega(t)=\left(\begin{array}{ccc}
\cos t & 0 & -\sin t \\
0 & \operatorname{Id}_{N-2} & 0 \\
\sin t & 0 & \cos t
\end{array}\right) .
$$

We consider the system $L=\partial_{t}+A(t) \partial_{x}$ with

$$
A(t)=\Omega(t) A_{1} \Omega^{-1}(t) .
$$

Note that $A(t)$ is an analytic function of $t$. For $v(t)=\Omega^{-1}(t) u(t)$, the equation $L u=f$ is transformed to

$$
\partial_{t} v+A_{1} \partial_{x} v+B v=\Omega^{-1} f
$$

where

$$
B=\Omega^{-1} \partial_{t} \Omega=\left(\begin{array}{ccc}
0 & 0 & -1 \\
0 & 0 & 0 \\
1 & 0 & 0
\end{array}\right) .
$$

Thus we are reduced to the perturbation of a constant coefficient nilpotent matrix, and we know that the optimal Gevrey index for the well posedness is $N /(N-1)$ : the eigenvalues of $i \xi A+B$ are the roots of

$$
\tau^{N}-(-i \xi)^{N-1}+\tau^{N-2}=0
$$

and their imaginary part grow like $c|\xi|^{(N-1) / N}$, with $c>0$. This implies Theorem 1.2.

\section{$3 \quad$ The general strategy for proving the well posed- ness}

Our analysis follows the original ideas of [CDGS, CJS, CoNi, Ta1] and relies on an energy method. We consider the Cauchy problem

$$
\partial_{t} u+\sum_{j=1}^{d} A_{j}(t) \partial_{x_{j}} u+B(t) u=f, \quad u_{\mid t=0}=h .
$$


The main part of the analysis consists in solving this equation when $f$ and $h$ have compact support in $x$. In this case, we perform a Fourier transform in the space variables. Denoting by $\hat{u}(t, \xi)$ the Fourier transform of $u$, we are reduced to solve:

$$
\partial_{t} \hat{u}+i A(t, \xi) \hat{u}+B(t) \hat{u}=\hat{f}, \quad \hat{u}_{\mid t=0}=\hat{h} .
$$

The main idea from [CDGS] is to use an energy method for this family of ordinary differential system depending on the parameter $\xi$. More precisely we aim to construct approximate symmetrizers $S(t, \xi)$ which have the following properties:

(S1) For $(t, \xi) \in[0, T] \times \mathbb{R}^{d}, S(t, \xi)$ is a self-adjoint positive definite matrix; moreover there is a positive scalar function $\Delta(t, \xi)$ and there are constants $C$ and $M$ such that for all $(t, \xi)$

$$
\begin{aligned}
& C^{-1} \Delta(t, \xi) \operatorname{Id} \leq S(t, \xi) \leq C \Delta(t, \xi) \mathrm{Id}, \\
& C^{-1}\langle\xi\rangle^{-M} \leq \Delta(t, \xi) \leq C\langle\xi\rangle^{M} .
\end{aligned}
$$

where we use the notation $\langle\xi\rangle=\left(1+|\xi|^{2}\right)^{\frac{1}{2}}$.

(S2) For all $\xi \in \mathbb{R}^{d}, S(\cdot, \xi)$ is absolutely continuous on $[0, T]$ and there is a function $\varphi(\cdot, \xi) \in L^{1}([0, T])$, a constant $C$ and $\alpha \in[0,1[$ such that

$$
\begin{aligned}
& \|\varphi(\cdot, \xi)\|_{L^{1}([0, T])} \leq C \\
& \partial_{t} S(t, \xi) \leq\langle\xi\rangle^{\alpha} \varphi(t, \xi) S(t, \xi) \quad \text { a.e. } t \in[0, T],
\end{aligned}
$$

(S3) There is a constant $C$ such that for all $(t, \xi) \in[0, T] \times \mathbb{R}^{d}$

$$
\operatorname{Im}(S(t, \xi) A(t, \xi)) \leq C\langle\xi\rangle^{\alpha} S(t, \xi) .
$$

Theorem 3.1. Suppose that there is a family of approximate symmetrizers which satisfy the properties (S1), (S2) and (S3) above. Suppose that $B \in$ $L^{\infty}([0, T])$. Then, for all index $s<1 / \alpha$, the Cauchy problem (3.1) is locally and globally well posed in $G^{s}$.

Proof. a) Estimates for the solutions of (3.2).

Fix $\xi \in \mathbb{R}^{d}$. For $f \in L^{1}\left([0, T], \mathbb{C}^{N}\right)$ and $h \in \mathbb{C}^{N}$ consider the solution $u \in C^{0}\left([0, T] ; \mathbb{C}^{N}\right)$ of the differential system

$$
\partial_{t} u+i A(t, \xi) u+B(t) u=f, \quad u_{\mid t=0}=h .
$$

The energy $\mathcal{E}(t)=(S(t, \xi) u(t), u(t))$ is in $W^{1,1}([0, T])$ and

$$
\left.\left.\partial_{t} \mathcal{E}=2 \operatorname{Re}(S u, f)+\left(\partial_{t} S u, u\right)+2(\operatorname{Im} S A u, u)\right)-2(\operatorname{Re} S B u, u)\right)
$$


For all $t$ and $\xi,(3.3)$ implies that

$$
|(S B u, u)| \leq C \Delta\|B\|_{L^{\infty}}|u|^{2} \leq C^{\prime}(S u, u) .
$$

Therefore

$$
\partial_{t} \mathcal{E} \leq 2 \operatorname{Re}(S u, f)+\left(C(\varphi(t, \xi)+1)\langle\xi\rangle^{\alpha}+C^{\prime}\right) \mathcal{E}(t) .
$$

This implies that there are constant $C_{0}, C_{1}$ and $C_{2}$ such that

$$
\begin{aligned}
(S(t, \xi) u(t), u(t))^{\frac{1}{2}} & \leq C_{0} e^{C_{1} \Phi(t)\langle\xi\rangle^{\alpha}+t C_{2}}(S(0, \xi) h, h)^{\frac{1}{2}} \\
& +C_{0} e^{C_{1} \Phi(t)\langle\xi\rangle^{\alpha}+t C_{2}} \int_{0}^{t}\left(S\left(t^{\prime}, \xi\right) f\left(t^{\prime}\right), f\left(t^{\prime}\right)\right)^{\frac{1}{2}} d t^{\prime}
\end{aligned}
$$

with

$$
\Phi(t)=\int_{0}^{t} \varphi\left(t^{\prime}\right) d t^{\prime}
$$

By (3.5) the function $\Phi$ are uniformly bounded and, using (3.3) and (3.4), we obtain that there is a constant $\gamma$ such that

$$
|u(t)| \leq C_{1}\langle\xi\rangle^{2 M} e^{\gamma\langle\xi\rangle^{\alpha}}\left(|h|+\int_{0}^{t}\left\|f\left(t^{\prime}\right)\right\| d t^{\prime}\right) .
$$

b) Existence of solutions for compactly supported data.

If $f$ and $h$ are compactly supported in $x$ are $G^{s}$ functions, there are $C$ and $\delta>0$ such that

$$
\forall(t, \xi), \quad|\hat{f}(t, \xi)| \leq C e^{-\delta\langle\xi\rangle^{1 / s}}, \quad|\hat{h}(\xi)| \leq C e^{-\delta|\xi|^{1 / s}},
$$

for some $\delta>0$ and finite $C$. By step a) the solutions $\hat{u}(\cdot, \xi)$ of the family of o.d.e. (3.2) satisfy

$$
\mid \hat{u}(t, \xi)) \mid \leq C_{1}\langle\xi\rangle^{2 M} e^{\gamma\langle\xi\rangle^{\alpha}} e^{-\delta\langle\xi\rangle^{1 / s}} .
$$

By assumption $\alpha<1 / s$, and thus for $\delta^{\prime}<\delta$ there is a constant $C_{2}$ such that

$$
|\hat{u}(t, \xi)| \leq C_{2} e^{-\delta^{\prime}|\xi|^{1 / s}} .
$$

This implies that $\hat{u}(t)$ is the Fourier transform of a function $u(t)$ of class $G^{s}$ in $x \in \mathbb{R}^{d}$, solution of $(3.1)$ on $[0, T] \times \mathbb{R}^{d}$. 
c) Propagation of the support.

Following [CDGS], we use the Paley-Wiener theorem to prove that the solutions found in step b) have compact support in $x$. Indeed, if $f$ and $h$ are supported in the ball $\{|x| \leq R\}$, their Fourier transform is an entire function of $\xi$ which satisfy for $(t, \xi) \in[0, T] \times \mathbb{C}^{d}$ :

$$
|\hat{f}(t, \xi)| \leq C e^{-\delta\langle\xi\rangle^{1 / s}} e^{R|\operatorname{Im} \xi|}, \quad|\hat{h}(\xi)| \leq C e^{-\delta|\xi|^{1 / s}} e^{R|\operatorname{Im} \xi|} .
$$

The solution of the o.d.e. (3.2) is defined for all $\xi \in \mathbb{C}^{d}$ and clearly holomorphic in $\xi$. We can estimate it using the symmetrizer $S(t, \operatorname{Re} \xi)$. There is a new term in the right hand side of $(3.9): \operatorname{Re}(S(t, \operatorname{Re} \xi) A(t, \operatorname{Im} \xi) u, u)$. By (3.3), we know that

$$
\begin{aligned}
|(S(t, \operatorname{Re} \xi) A(t, \operatorname{Im} \xi) u, u)| & \leq C \Delta(t, \operatorname{Re} \xi)|\operatorname{Im} \xi||u|^{2} \\
& \leq \gamma_{1}|\operatorname{Im} \xi|(S(t, \operatorname{Re} \xi) u, u) .
\end{aligned}
$$

Continuing as in step a), instead of (3.13) we obtain that for $\xi \in \mathbb{C}^{d}$,

$$
\mid \hat{u}(t, \xi)) \mid \leq C_{1}\langle\xi\rangle^{2 M} e^{\gamma\langle\xi\rangle^{\alpha}} e^{\left(R+\gamma_{1} t\right)|\operatorname{Im} \xi|} e^{-\delta\langle\xi\rangle^{1 / s}} .
$$

By Paley-Wiener theorem, this implies that $u(t, \cdot)$ is supported in the ball $\left\{|x| \leq R+\gamma_{1} t\right\}$.

d) Local uniqueness and end of the proof of the theorem.

By classical duality arguments, solving the backward Cauchy problem for data with compact support, one obtains the local uniqueness of solutions of (3.1), even in spaces of ultra-distributions $\left(G^{s}\right)^{\prime}$.

When $f \in C^{0}\left([0, T], G^{s}\right)$ and $h \in G^{s}$, we can split them into locally finite sums of compactly supported functions, using a $G^{s}$ partition of unity. We can solve the Cauchy Problem for each piece by step a), and glue the pieces together using the finite speed of propagation of step c) and the local uniqueness result.

Remark 3.2. The constant $\gamma=\gamma(T)$ in (3.13) is estimated by

$$
C \sup _{\xi} \int_{0}^{T} \varphi(t, \xi) d t
$$

Therefore, for the critical exponent $s_{0}=1 / \alpha$, the proof above would provide a local in time solution on $[0, T] \times \mathbb{R}^{d}$ in $G^{s_{0}}$, as long as $\gamma=\gamma(T)<\delta$. It is not clear that this can be achieved simply by choosing $T$ small because we do not know wether the $\varphi(\cdot, \xi)$ are uniformly integrable or not.

Remark 3.3. Of course, when $\alpha=0$, there is no exponential loss in (3.13), and the Cauchy problem is well posed in $C^{\infty}$. 


\section{Colombini-Jannelli-Spagnolo-Tarama's lemma and extensions}

In [Ta1], S.Tarama proved the following extension of a result obtained in [CJS] for nonnegative functions.

Lemma 4.1. Given $0<\mu \leq 1$ and $k+\mu \geq 1$, there is a constant $C$ such that for all $a \in C^{k, \mu}([0, T])$ and $\delta>0$ :

$$
\int_{0}^{T} \frac{\left|\partial_{t} a(t)\right|}{(|a(t)|+\delta)^{1-1 /(k+\mu)}} d t \leq C\|a\|_{C^{k, \mu}}^{1 /(k+\mu)}
$$

This can be extended to $\delta=0$ and to functions with values in $\mathbb{R}^{n}$ in the following way.

Proposition 4.2. Given $0<\mu \leq 1$ and $k+\mu \geq 1$, there is a constant $C$ such that for all $a \in C^{k, \mu}\left([0, T], \mathbb{R}^{n}\right)$, there is a nonnegative function $a^{\sharp}$ in $L^{1}([0, T])$ such that for almost all $t \in[0, T]$

$$
\left|\partial_{t} a(t)\right| \leq a^{\sharp}(t)|a(t)|^{1-1 /(k+\mu)},
$$

and

$$
\left\|a^{\sharp}\right\|_{L^{1}([0, T])} \leq C\|a\|_{C^{k, \mu}}^{1 /(k+\mu)}
$$

Proof. Consider first the scalar case $n=1$. The sequence

$$
\frac{\left|\partial_{t} a(t)\right|}{(|a(t)|+1 / j)^{1-1 /(k+\mu)}}
$$

is nondecreasing in $j$, hence by Beppo Levi's lemma its limit $a^{\sharp}(t) \in[0,+\infty]$ satisfies

$$
\int_{0}^{T} a^{\sharp}(t) d t=\lim _{j \rightarrow \infty} \int_{0}^{T} \frac{\left|\partial_{t} a(t)\right|}{(|a(t)|+1 / j)^{1-1 /(k+\mu)}} d t \leq C
$$

and hence $a^{\sharp} \in L^{1}([0, T])$. Moreover, for all $t$ and $j$

$$
\left|\partial_{t} a(t)\right| \leq a^{\sharp}(t)(|a(t)|+1 / j)^{1-1 /(k+\mu)} .
$$

One can pass to the limit at every point where $a^{\sharp}$ is finite, that is almost everywhere, and thus (4.2) follows. 
If $a=\left(a_{1}, \ldots, a_{n}\right)$ takes its values in $\mathbb{R}^{n}$, we can find functions $a_{j}^{\sharp}$ in $L^{1}$ such that the estimate (4.2) is satisfied for all $j$. Choosing $a^{\sharp}$ of the form $C \sum a_{j}^{\#}$, the proposition follows.

Remark 4.3. On the open set $\{a \neq 0\}$, the construction above gives

$$
a^{\sharp}(t)=\frac{\left|\partial_{t} a(t)\right|}{|a(t)|^{1-1 /(k+\mu)}} .
$$

On the set $\left\{a(t)=\partial_{t} a(t)=0\right\}$, the inequality (4.2) is satisfied for arbitrary finite $a^{\sharp}(t)$, and for instance we can choose $a^{\sharp}(t)=0$. The set $\left\{a=0, \partial_{t} a \neq\right.$ $0\}$ has Lebesgue measure equal to 0 , since it is the union of the finite sets $\left\{a=0,\left|\partial_{t} a\right| \geq 1 / j\right\}$. Therefore, we can define $a^{\sharp}$ by (4.4) when $a(t) \neq 0$, and by 0 elsewhere.

We will use the following corollary of Proposition 4.2

Proposition 4.4. Given $0<\mu \leq 1$ and $k+\mu \geq 1$, there is a constant $C$ such that for all $a \in C^{k, \mu}\left([0, T], \mathbb{R}^{n}\right), \Delta \in C^{0}\left([0, T] ; \mathbb{R}_{+}\right), \delta>0$ satisfying

$$
\forall t \in[0, T], \quad|a(t)| \leq \Delta(t) \quad \text { and } \quad \delta \leq \Delta(t),
$$

the function $\varphi(t)=\left|\partial_{t} a(t)\right| / \Delta(t)$ satisfies

$$
\|\varphi\|_{L^{1}([0, T])} \leq C \delta^{-1 /(k+\mu)}\|a\|_{C^{k, \mu}}^{1 /(k+\mu)} .
$$

Proof. By Proposition 4.2, one has

$$
\left|\partial_{t} a(t)\right| \leq a^{\sharp}(t)|a(t)|^{1-1 /(k+\mu)} \leq a^{\sharp}(t) \Delta^{1-1 /(k+\mu)} \leq a^{\sharp}(t) \delta^{-1 /(k+\mu)} \Delta(t)
$$

and the proposition follows.

\section{Proof of Theorem $\mathbf{1 . 6}$}

In this section we consider $2 \times 2$ systems and more generally systems with eigenvalues of multiplicity at most two.

\subsection{Symmetrizers for $2 \times 2$ systems}

Consider general $2 \times 2$ matrices

$$
A=\lambda \mathrm{Id}+A^{\prime}, \quad A^{\prime}=\left(\begin{array}{cc}
a & b \\
c & -a
\end{array}\right), \quad \lambda=\frac{1}{2} \operatorname{tr} A .
$$


This matrix is hyperbolic, that is has real eigenvalues, when $\lambda$ is real and $h:=a^{2}+b c$ is real and nonnegative. A set of such matrices is uniformly diagonalizable if there is a positive real number $\delta$, such that for all matrices in this set:

$$
h \geq \delta\left|A^{\prime}\right|^{2}
$$

Because $A^{\prime 2}=h \mathrm{Id}$, an exact symmetrizer of $A^{\prime}$, and thus of $A$, is

$$
S=A^{*} A^{\prime}+h \mathrm{Id} .
$$

It is self adjoint and non negative since

$$
(S u, u)=\left|A^{\prime} u\right|^{2}+h|u|^{2} .
$$

Moreover, for matrices which satisfy (5.2), one has

$$
h|u|^{2} \leq(S u, u)=(1+1 / \delta) h|u|^{2} .
$$

The symmetrizer $S$ is not definite positive when $h=0$, that is when $A$ has a multiple (double) eigenvalue. Following the ideas of [CDGS, CJS], one adds a (small) corrector to $S$ and consider for $\varepsilon>0$ :

$$
S_{\varepsilon}=S+\varepsilon^{\varepsilon} \operatorname{Id}=A^{*} A^{\prime}+\left(h+\varepsilon^{2}\right) \operatorname{Id} .
$$

If the condition (5.2) is satisfied, then, with $h_{\varepsilon}=h+\varepsilon^{2}>0$

$$
\varepsilon|u|^{2} \leq h_{\varepsilon}|u|^{2} \leq(S u, u)=(1+1 / \delta) h_{\varepsilon}|u|^{2} .
$$

Moreover, $\operatorname{Im} S_{\varepsilon} A=\varepsilon^{2} \operatorname{Im} A^{\prime}$. Therefore, for matrices which satisfy (5.2), one has

$$
\left|\left(\operatorname{Im} S_{\varepsilon} A u, u\right)\right| \leq \varepsilon^{2}(h / \delta)^{\frac{1}{2}}|u|^{2} \leq \delta^{-\frac{1}{2}} \varepsilon h_{\varepsilon}|u|^{2} \leq \delta^{-\frac{1}{2}} \varepsilon\left(S_{\varepsilon} u, u\right),
$$

where we have used that $\varepsilon h^{\frac{1}{2}} \leq h+\varepsilon^{2}=h_{\varepsilon}$.

If the matrices $A(t)$ are $C^{k, \mu}$ functions of $t \in[0, T]$, the explicit form (5.3) shows that $S(t)$ and $S_{\varepsilon}(t)$ are $C^{k, \mu}$ functions of $t$. Moreover, Proposition 4.2 implies that there is a constant $C_{0}$ and a function $\varphi \in L^{1}([0, T])$ such that for all $C^{k, \mu}$ matrix $A^{\prime}(t)$ :

$$
\left|\partial_{t} A^{\prime}(t)\right| \leq \varphi(t)\left|A^{\prime}(t)\right|^{1-1 /(k+\mu)}
$$

and

$$
\|\varphi\|_{L^{1}([0, T])} \leq C_{0}\|A\|_{C^{k, \mu}}^{1 / k+\mu} .
$$


Therefore, if the matrices $A(t)$ satisfy (5.2), one has

$$
\left|\partial_{t} S(t)\right| \lesssim\left|A^{\prime}(t)\right|\left|\partial_{t} A^{\prime}(t)\right| \lesssim \varphi(t) h(t)^{1-1 / 2 k} \lesssim \varphi(t) \varepsilon^{-1 / k} h_{\varepsilon}(t)
$$

and thus

$$
\left|\left(\partial_{t} S_{\varepsilon}(t) u, u\right)\right| \leq C_{1} \varphi(t) \varepsilon^{-1 / k}\left(S_{\varepsilon}(t) u, u\right)
$$

where $C_{1}$ depends only on $k, \mu$ and the constant $\delta$ in (5.2).

Similarly, since $h$ is quadratic in the coefficients of $A^{\prime}$, one has

$$
\left|\partial_{t} h(t)\right| \lesssim \varphi(t) h(t)^{1-1 / 2(k+\mu)} \lesssim \varphi(t) \varepsilon^{-1 /(k+\mu)} h_{\varepsilon}(t) .
$$

Summing up we have proved the following:

Proposition 5.1. There is a constant $C_{0}$ and, given $\delta>0$, there is another constant $C_{1}$, such that: for all $C^{k+\mu}$ family of hyperbolic matrices $A(t)$ which satisfy (5.2), the approximate symmetrizers $S_{\varepsilon}(t)$ defined by (5.5) for $\varepsilon \in$ ] $0,1]$, satisfy the properties (5.4), (5.7) and there is a function $\varphi \in L^{1}([0, T])$ satisfying (5.8) such that the inequalities (5.9) and (5.10) hold for almost all $t$.

The important point in this proposition, is that all the estimates are uniform in $A$ satisfying (5.2) and bounded in $C^{k+\mu}([0, T])$.

\subsection{Symmetrizers for $2 \times 2$ systems}

Proposition 5.2. Consider a $2 \times 2$ matrix $A(t, \xi)$, homogeneous of degree one in $\xi$. We assume that it is uniformly diagonalizable and that its coefficients are bounded in $C^{k, \mu}([0, T])$ for $|\xi|=1$, with $k \geq 1$. Then, there is a family of approximate symmetrizers $S(t, \xi)$ which satisfy the properties (S1) (S2) and (S3) listed before Theorem 3.1, with parameter $\alpha=1 /(k+\mu+1)$.

Proof. The statement is trivial when $|\xi| \leq 1$, so we only consider the case $|\xi| \geq 1$. It is convenient to use polar coordinates $\xi=\rho \omega$ with $\rho=|\xi| \geq 1$. We use the symmetrizers $S_{\varepsilon}(t, \omega)$ associated to the matrices $A(t, \omega)$. They satisfy the estimates (5.6), (5.7) and (5.9) with constants independent of $\omega$, with functions $\varphi(\cdot, \omega)$ uniformly bounded in $L^{1}$.

Thus

$$
\left|\partial_{t} S_{\varepsilon}\right| \lesssim \varphi \varepsilon^{-1 /(k+\mu)} S_{\varepsilon}, \quad \operatorname{Im} S_{\varepsilon} A(t, \xi) \lesssim \varepsilon|\xi| S_{\varepsilon}
$$

Now we choose $\varepsilon=|\xi|^{-(k+\mu) /(k+\mu+1)}$ to balance the two terms $\varepsilon^{-1 /(k+\mu)}$ and $\varepsilon|\xi|$, and define

$$
S(t, \xi)=S_{\varepsilon}(t, \omega), \quad \omega=\frac{\xi}{|\xi|}, \quad \varepsilon=|\xi|^{-k /(k+1)} .
$$


It satisfies the conditions (S2) and (S3) with $\alpha=1 /(k+\mu+1)$. With this choice $h(t, \xi)=h_{\varepsilon}(t, \omega)$ satisfies

$$
|\xi|^{-2} \leq|\xi|^{-2 k /(k+1)} \leq h(t, \xi) \leq C
$$

and there is a constant $C$ such that

$$
C^{-1} h(t, \xi) \operatorname{Id} \leq S(t, \xi) \leq C h(t, \xi) \operatorname{Id} .
$$

which means that the condition (S1) is also satisfied. For further use, we note that

$$
\left|\partial_{t} h(t, \xi)\right| \leq C \varphi(t) \varepsilon^{-1 /(k+\mu)} h(t, \xi) \leq C \varphi(t)|\xi|^{1 /(k+\mu+1)} h(t, \xi) .
$$

as a consequence of (5.10).

\subsection{Symmetrizers for systems with multiplicities at most 2}

Consider a $N \times N$ system (3.1), uniformly diagonalizable, with coefficients $A_{j} \in C^{k+\mu}([0, T])$ with $k \geq 1$. The eigenvalues of $A(t, \xi)$ can be labelled in increasing order $\lambda_{1}(t, \xi) \leq \ldots \leq \lambda_{N}(t, \xi)$. We make the following assumption.

Assumption 5.3. For all $j$ and $\left(t_{0}, \xi_{0}\right) \in[0, T] \times \mathbb{R}^{d} \backslash\{0\}$, either the multiplicity of $\lambda_{j}(t, \xi)$ is constant on a neighborhood of $\left(t_{0}, \xi_{0}\right)$, or it is less than or equal to two.

Proposition 5.2 has the following extension, which, together with Theorem 3.1, finishes the proof of Theorem 1.6.

Theorem 5.4. Under the assumptions above, there is a family of approximate symmetrizers $S(t, \xi)$ which satisfy the properties (S1) (S2) and (S3) with parameter $\alpha=1 /(k+\mu+1)$. Hence, for all $B \in C^{0}([0, T])$, the Cauchy problem (3.1) is locally and globally well posed in $G^{s}$ for $s<1+k+\mu$.

Proof. We consider again the system on the Fourier side, for $|\xi| \geq 1$. By finite partition of unity, it is sufficient to prove that for all $\left(t_{0}, \xi_{0}\right)$ with $\left|\xi_{0}\right|=1$, one can construct a symmetrizer with the desired properties on a conical neighborhood of this point in $[0, T] \times \mathbb{R}^{d} \backslash\{0\}$.

Given $\left(t_{0}, \xi_{0}\right)$, there is an invertible matrix $P(t, \xi)$ defined on a conical neighborhood of this point, homogeneous of degree 0 in $\xi, C^{k+\mu}$ in $t$, such that

$$
\tilde{A}(t, \xi):=P(t, \xi) A(t, \xi) P^{-1}(t, \xi)=\operatorname{diag}\left(A_{0}(t, \xi), \ldots, A_{m}(t, \xi)\right)
$$


is block diagonal, with

i) $A_{0}$ has only eigenvalues of constant multiplicity,

ii) $A_{1}, \ldots, A_{m}$ are $2 \times 2$ matrices.

There is an exact $C^{k, \mu}$ symmetrizer $S_{0}(t, \xi)$ for $A_{0}(t, \xi)$, satisfying

$$
C^{-1} \mathrm{Id} \leq S_{0} \leq C, \quad \partial_{t} S_{0} \leq C S_{0}, \quad \operatorname{Im} S_{0} A_{0}=0 .
$$

On the given conical neighborhood, the $2 \times 2$ block $A_{j}$ have approximate symmetrizers given by Proposition 5.2. They satisfy

$$
\left\{\begin{array}{l}
\partial_{t} S_{j}(t, \xi) \leq\langle\xi\rangle^{1 /(k+\mu+1)} \varphi_{j}(t, \xi) S_{j}(t, \xi), \quad\left\|\varphi_{j}(\cdot, \xi)\right\|_{L^{1}} \leq C, \\
\operatorname{Im} S_{j}(t, \xi) \tilde{A}_{j}(t, \xi) \leq C|\xi|^{1 /(k+\mu+1)} S_{j}(t, \xi), \\
C^{-1} h_{j}(t, \xi) \operatorname{Id} \leq S_{j}(t, \xi) \leq C h_{j}(t, \xi) \operatorname{Id},
\end{array}\right.
$$

where the $h_{j}$ satisfy

$$
\left\{\begin{array}{l}
|\xi|^{-2} \leq h_{j}(t, \xi) \leq C \\
\left|\partial_{t} h_{j}(t, \xi)\right| \leq C \varphi_{j}(t)|\xi|^{1 /(k+\mu+1)} h_{j}(t, \xi) .
\end{array}\right.
$$

The first candidate to symmetrize $\tilde{A}$ would be $\operatorname{diag}\left(A_{0}(t, \xi), \ldots, A_{m}(t, \xi)\right)$ but it has not necessarily the property (S1) since the $h_{j}$ are different. This can be corrected in the following way. Introduce

$$
\Delta(t, \xi)=\prod_{j=1}^{m} h_{j}(t, \xi)
$$

and for $j \geq 1$

$$
\Delta_{j}(t, \xi)=\prod_{l \neq j} h_{l}(t, \xi)
$$

Consider the symmetrizer

$$
\tilde{S}(t, \xi)=\operatorname{diag}\left(\Delta A_{0}, \Delta_{1} S_{1}, \ldots, \Delta_{m} S_{m}\right) .
$$

Each block is a symmetric matrix $\approx \Delta \mathrm{Id}$, and thus there is $C$ such that

$$
C^{-1} \Delta(t, \xi) \operatorname{Id} \leq \tilde{S}(t, \xi) \leq C \Delta(t, \xi) \mathrm{Id},
$$

while $\Delta$ satisfies

$$
|\xi|^{-2 m} \leq \Delta(t, \xi) \leq C
$$


By (5.17), one has

$$
\partial_{t} \Delta \leq C \varphi|\xi|^{1 /(k+\mu+1)} \Delta, \quad \partial_{t} \Delta_{j} \leq C \varphi|\xi|^{1 /(k+\mu+1)} \Delta_{j}
$$

with $\varphi=1+\sum \varphi_{j}$. With (5.16), this implies that

$$
\left|\partial_{t} \tilde{S}(t, \xi)\right| \leq C \varphi(t, \xi)|\xi|^{1 /(k+\mu+1)} \tilde{S}(t, \xi) .
$$

The scalar factors $\Delta_{j}$ commute to the block $A_{j}$ and therefore

$$
\operatorname{Im}(\tilde{S}(t, \xi) \tilde{A}(t, \xi)) \leq C|\xi|^{1 /(k+1)} \tilde{S}(t, \xi) .
$$

Hence $\tilde{S}$ satisfies the properties (S1) (S2) and (S3) for $\tilde{A}$.

Let

$$
S(t, \xi)=P^{*}(t, \xi) \tilde{S}(t, \xi) P(t, \xi) .
$$

The properties (S1) and (S3) are immediately transported from $\tilde{S}$ to $S$. Next, we have

$$
\partial_{t} S=P^{*} \partial_{t} \tilde{S} P+\partial_{t} P^{*} \tilde{S} P+P^{*} \tilde{S} \partial_{t} P .
$$

By (5.21), the first term is $O\left(\varphi|\xi|^{1 /(k+\mu+1)} S\right)$. By (5.19), the second and third terms are $O(\Delta)=O(S)$. This shows that $S$ also has the property (S2), which finishes the proof of the theorem.

\section{Construction of symmetrizers}

\subsection{Preliminary remarks}

A possible definition of approximate symmetrizers for a hyperbolic matrix $A$, that is a matrix with only real eigenvalues, is

$$
\Sigma_{\varepsilon}=2 \varepsilon \int_{0}^{\infty} e^{i s A^{*}} e^{-i s A} e^{-s \varepsilon} d s=2 \varepsilon \int_{0}^{\infty} e^{i s M^{*}} e^{-i s M} d s
$$

with $M=A-\frac{i}{2} \varepsilon \mathrm{Id}$. When $A$ is diagonalizable, $A=\sum \lambda_{j} \Pi_{j}$ and

$$
\Sigma_{\varepsilon} \rightarrow \Sigma_{0}=2 \sum \Pi_{j}^{*} \Pi_{j} .
$$

If $\mathcal{K}$ is a compact set of uniformly diagonalizable matrices, there is a constant $C$ such that for $A \in \mathcal{K}$ and $s \in \mathbb{R}$, one has $\left|e^{-i s A} u\right| \leq C|u|$. Reversing time, this implies that $|u| \leq C\left|e^{-i t A} u\right|$ and therefore there is a constant $C$ such that

$$
A \in \mathcal{K}, \varepsilon \geq 0 \Rightarrow C^{-1}|u|^{2} \leq\left(\Sigma_{\varepsilon} u, u\right) \leq C|u|^{2} .
$$


If $A$ is a Lipschitz function of $t$ with values in $\mathcal{K}$, differentiating in time the o.d.e $\partial_{s} u+i A(t) u=0$ yields the estimate

$$
\mid \partial_{t}\left(e^{-i s A(t)}|\leq C s| \partial_{t} A(t) \mid\right.
$$

which implies that

$$
\left|\partial_{t} \Sigma_{\varepsilon}\right| \leq C \varepsilon^{-1}
$$

As mentionned in the introduction the proofs of Theorems 1.4 and 1.6 rely on an improvement of this estimate, and this is clearly what we did in Section 5 for $2 \times 2$ systems. In general, that is if the multiplicities are not constant, there is no hope that $\partial_{t} \Sigma_{\varepsilon}$ remains bounded as $\varepsilon \rightarrow 0$. Our goal is to replace $\Sigma_{\varepsilon}$ by symmetrizers $S_{\varepsilon}$ which are smooth functions of the coefficients of the matrix $A$, up to $\varepsilon=0$. The basic remark is the following.

Lemma 6.1. There is a polynomial $\Delta$ of $\varepsilon$ and the coefficients of $A$ and $A^{*}$, such that the coefficients of $\Delta \Sigma_{\varepsilon}$ are polynomials of $\varepsilon$ and the coefficients of $A$ and $A^{*}$.

Proof. We note that $\Sigma_{\varepsilon}$ satisfies

$$
\operatorname{Im}\left(\Sigma_{\varepsilon} A\right)=\frac{1}{2} \varepsilon \Sigma_{\varepsilon}-\varepsilon \operatorname{Id}, \quad \operatorname{Im}\left(\Sigma_{\varepsilon} M\right)=-\varepsilon \operatorname{Id} .
$$

Denote by $\mathcal{M}$ the mapping $\Sigma \mapsto \Sigma M-M^{*} \Sigma$. Because $M$ and $M^{*}$ have no common eigenvalue, this mapping is a bijection on the space of $N \times N$ matrices, and $\Sigma_{\varepsilon}$ is the solution of

$$
\mathcal{M}\left(\Sigma_{\varepsilon}\right)=-2 i \mathrm{Id}
$$

By uniqueness, the solution of this equation is self adjoint, and we could have defined $\Sigma_{\varepsilon}$ as the solution of (6.6). The determinant $\Delta$ of $\mathcal{M}$ is a polynomial of $\varepsilon$ and the coefficients of $A$ and $A^{*}$, and Cramer's formula for the solution of (6.6) imply the Lemma.

The polynomials involved in this result are identified in the next subsection using a different approach which is motivated by the following remark: if one writes $\Delta \mathcal{M}^{-1}$ as a polynomial of $\mathcal{M}$ and if one notes that $\mathcal{M}^{n}(\Sigma)$ is a linear combination of $M^{* k} \Sigma M^{j}$ with $k+j=n$, one obtains that $\Delta \Sigma_{\varepsilon}$ has the form

$$
\Delta \Sigma_{\varepsilon}=\sum \sigma_{k, l} M^{* l} M^{l}
$$




\subsection{The new approach}

We start from expressions of the form (6.7), which extends (5.3). Changing the labeling for convenience, they are associated to polynomials

$$
S(X, Y)=\sum_{k=0}^{m-1} \sum_{l=0}^{m-1} \sigma_{k, l} X^{m-1-k} Y^{m-1-l} .
$$

Given such a polynomial and a matrix $M$ we define

$$
S\left(M^{*}, M\right):=\sum_{k=0}^{m-1} \sum_{l=0}^{m-1} \sigma_{k, l} M^{* m-1-k} M^{m-1-l} .
$$

The symmetry condition reads

$$
S(Y, X)=\bar{S}(X, Y)
$$

where, in general, given a polynomial $P$, we denote by $\bar{P}$ the polynomial whose coefficients are the complex conjugate of those of $P$. This condition implies that $\bar{\sigma}_{k, l}=\sigma_{l, k}$ and thus, for all $M$, that $S\left(M^{*}, M\right)$ is self adjoint.

Lemma 6.2. Let $S(X, Y)$ be a polynomial (6.8) satisfying (6.10), and let $T(X, Y)=-\frac{1}{2 i}(X-Y) S(X, Y)$. Then for all matrix $M$,

$$
\operatorname{Im}\left(S\left(M^{*}, M\right) M\right)=T\left(M^{*}, M\right) .
$$

Proof. Because the variables $M^{*}$ and $M$ do not commute, one has to be careful and we include a proof. The definition of $T$ is that its coefficients $\tau_{k, l}$ are

$$
\tau_{k, l}=-\frac{1}{2 i}\left(\sigma_{k-1, l}-\sigma_{k, l-1}\right)
$$

with the convention that $\sigma_{k, l}=0$ if $k<0$ or $l<0$. Because $S=S\left(M^{*}, M\right)$ is self adjoint, one has

$$
\begin{aligned}
\operatorname{Im}(S M) & =\frac{1}{2 i}\left(S M-M^{*} S\right)=\frac{1}{2 i} \sum \sigma_{k, l}\left(M^{* k} M^{l+1}-M^{* k+1} M^{l}\right) \\
& =\frac{1}{2 i} \sum\left(\sigma_{k, l-1}-\sigma_{k-1, l}\right) M^{* k} M^{l}=T\left(M^{*}, M\right)
\end{aligned}
$$

as claimed.

Thus, to obtain symmetrizers for a hyperbolic matrix $A$, it is sufficient to consider polynomials $S(X, Y)$ such that $(X-Y) S(X, Y)$ can be factorized by the characteristic polynomial $P_{A}(Y)$ of $A$ on the right, and by the characteristic polynomial, $\bar{P}_{A}(X)=P_{A}(X)$, of $M^{*}$ on the left. 
Example 6.3. Suppose that the characteristic polynomial $P_{A}$ of $A$ has real coefficients. For any polynomial $Q$ with real coefficients one can define the polynomial

$$
S(X, Y)=\frac{P_{A}(X) Q(Y)-Q(X) P_{A}(Y)}{X-Y}
$$

Then $S\left(A^{*}, A\right)$ is self adjoint. Using Lemma 6.2 and the identity $P_{A}(A)=$ $P_{A}\left(A^{*}\right)=0$, we see that $\operatorname{Im} S\left(A^{*}, A\right) A=0$.

One looks for approximate symmetrizers for $M=A-i \frac{1}{2} \mathrm{Id}$, which satisfy

$$
\operatorname{Im}(S M)=-\varepsilon \Delta \operatorname{Id}
$$

were $\Delta$ is real and positive. Denote by $P_{M}$ the characteristic polynomial of $M$. If $Q$ is a polynomial such that

$$
\bar{P}_{M}(X) Q(X)-\bar{Q}(X) P_{M}(X)+2 i \varepsilon \Delta=0,
$$

then

$$
S(X, Y)=\frac{\bar{P}_{M}(X) Q(Y)-\bar{Q}(X) P_{M}(Y)+2 i \varepsilon \Delta}{X-Y},
$$

(6.13) is a polynomial and Lemma 6.2 implies that $S\left(M^{*}, M\right)$ is a solution of $(6.11)$.

Remark 6.4. Because $P_{M}(M)=\bar{P}_{M}\left(M^{*}\right)=0$, it is sufficient to consider polynomials in $\mathbb{R}(X) / P_{M}$. In particular, we can bound the degree $m-1$ in (6.9) by $N-1$, where $N$ is the dimension of the matrix.

\subsection{Positive approximate symmetrizers for hyperbolic ma- trices}

Consider a $N \times N$ hyperbolic matrix $A$ with real eigenvalues $\lambda_{j}$ and denote by $P_{A}$ its characteristic polynomial:

$$
P_{A}(X)=X^{N}+\sum_{k=1}^{N} p_{k} X^{N-k}=\prod_{j=1}^{N}\left(X-\lambda_{j}\right) .
$$

The characteristic polynomial $P_{M}$ of $M=A-\frac{i}{2} \varepsilon \mathrm{Id}$ is

$$
P_{M}(X)=p_{A}(X+i \varepsilon / 2)=\prod_{j=1}^{N}\left(X-\lambda_{j}+i \varepsilon / 2\right) .
$$


The condition (6.12) leads to take $\varepsilon \Delta$ to be equal (up to a factor) to the resultant of $P_{M}$ and $\bar{P}_{M}$, which is

$$
\begin{aligned}
\operatorname{Res}\left(P_{M}, \bar{P}_{M}\right) & =\prod_{j, k}\left(\lambda_{j}-\lambda_{k}-i \varepsilon\right) \\
& =(-2 i \varepsilon)^{N}(-1)^{N(N-1) / 2} \prod_{j<k}\left(\left|\lambda_{j}-\lambda_{k}\right|^{2}+\varepsilon^{2}\right) .
\end{aligned}
$$

There are too many factors $\varepsilon$, which can be eliminated as follows. Introduce the polynomial $R$ such that

$$
P_{A}\left(X-i \frac{1}{2} \varepsilon\right)-P_{A}\left(X+i \frac{1}{2} \varepsilon\right)=-i \varepsilon R(X, \varepsilon) .
$$

$R$ has real coefficients, and denoting by $R_{\varepsilon}(X)=R(X, \varepsilon)$, we have

$$
\begin{aligned}
\operatorname{Res}\left(P_{M}, \bar{P}_{M}\right) & =\operatorname{Res}\left(P_{M}, P_{M}-i \varepsilon R_{\varepsilon}\right) \\
& =\operatorname{Res}\left(P_{M},-i \varepsilon R_{\varepsilon}\right)=(-i \varepsilon)^{N} \operatorname{Res}\left(P_{M}, R_{\varepsilon}\right) .
\end{aligned}
$$

Comparing with $(6.16)$, this shows that $\operatorname{Res}\left(P_{M}, R_{\varepsilon}\right)=(-1)^{N(N-1) / 2} \Delta_{\varepsilon}$ where

$$
\Delta_{\varepsilon}=\prod_{j<k}\left(\left|\lambda_{j}-\lambda_{k}\right|^{2}+\varepsilon^{2}\right) .
$$

We note that $\Delta_{\varepsilon}$ is a polynomial in $\varepsilon$ and the coefficients $p_{1}, \ldots, p_{m}$, and thus a polynomial of $\varepsilon$ and the coefficients $\left[a_{j, k}\right]$ of the matrix $A$.

There are $Q$ and $Q_{1}$, polynomials with coefficients in $\mathbb{Z}$ of $X, \varepsilon$ and the coefficients of $P_{M}$ and $R_{\varepsilon}$, thus polynomials of $X, \varepsilon$ and the coefficients $\left[a_{j, k}\right]$, such that

$$
P_{M}(X) Q_{1}(X)+R_{\varepsilon} Q(X)=2 \Delta_{\varepsilon}
$$

Therefore

$$
2 i \varepsilon \Delta_{\varepsilon}=P_{M}\left(Q+i \varepsilon Q_{1}\right)-\bar{P}_{M} Q
$$

Because $\Delta_{\varepsilon}$ is real:

$$
-2 i \varepsilon \Delta_{\varepsilon}=\bar{P}_{M}\left(\bar{Q}-i \varepsilon \bar{Q}_{1}\right)-P_{M} \bar{Q}
$$

and by uniqueness of the decomposition when $\Delta \neq 0$, this implies that $\bar{Q}-i \varepsilon \bar{Q}_{1}=Q$ and hence

$$
\bar{P}_{M}(X) Q(X)-\bar{Q}(X) P_{M}(X)+2 i \varepsilon \Delta_{\varepsilon}=0 .
$$

Summing up, we have proved the following: 
Lemma 6.5. There is a polynomial $Q\left(X, \varepsilon,\left[a_{j, k}\right]\right)$, such that for all hyperbolic matrix A the identity (6.20) is satisfied.

Corollary 6.6. There is a polynomial $\Delta$ of $\left(\varepsilon,\left[a_{j, k}\right]\right)$ and there is a polynomial $S$, of $\left(X, Y, \varepsilon,\left[a_{j, k}\right]\right)$, such that for all hyperbolic matrix $A$ with coefficients $\left[a_{j, k}\right], \Delta\left(\varepsilon,\left[a_{j, k}\right]\right)$ is given by $(6.18)$ and

$$
S_{\varepsilon}=S\left(A^{*}+i \varepsilon / 2, A-i \varepsilon / 2, \varepsilon,\left[a_{j, k}\right]\right)
$$

is a symmetric matrix such that

$$
\operatorname{Im} S_{\varepsilon} A=\frac{1}{2} \varepsilon S-\varepsilon \Delta_{\varepsilon} \operatorname{Id} .
$$

Moreover, for $\varepsilon>0, S_{\varepsilon}=\Delta_{\varepsilon} \Sigma_{\varepsilon}$ where $\Sigma_{\varepsilon}$ is given by (6.1).

Proof. It only remains to prove the last statement. $S=S_{\varepsilon}-\Delta_{\varepsilon} \Sigma_{\varepsilon}$ satisfies $2 i \operatorname{Im}(S M)=S M-M^{*} S=0$. For $\varepsilon>0, M$ and $M^{*}$ have no common eigenvalue and thus $S=0$.

Remark 6.7. Because everything is polynomial, we can take $\varepsilon=0$ in the construction above. In this case, $P_{M}=P_{A}, R_{0}=P^{\prime}, \Delta_{0}=\Delta$ is the discriminant of $P_{A}$ and $S_{0}$ is an exact symmetrizer of $A$. Moreover, if $A$ is diagonalizable, then

$$
S\left(A^{*}, A\right)=2 \Delta(P) \sum \Pi_{k}^{*} \Pi_{k}
$$

where $A=\sum \lambda_{k} \Pi_{k}$ is the spectral decomposition of $A$. This implies in particular that, on the set of strictly hyperbolic matrices, the symmetrizer $\sum \Pi_{k}^{*} \Pi_{k}$ is a rational function of the coefficients of $A$.

Remark 6.8. The construction can be made using the minimal polynomial of $A$ in place of the characteristic polynomial $P_{A}$. This makes sense, if $A$ is restricted to a set where this minimal polynomial is a smooth function of the coefficients of $A$.

The next result follows directly from (6.3).

Proposition 6.9. Let $\mathcal{K}$ denote a set of uniformly diagonalizable hyperbolic matrices. Then there is a constant $C$ such that for all $A \in \mathcal{K}$ and all $\varepsilon \geq 0$ the symmetrizers constructed above satisfy

$$
C^{-1} \Delta_{\varepsilon} \mathrm{Id} \leq S_{\varepsilon} \leq C \Delta_{\varepsilon} \mathrm{Id}
$$




\section{Applications}

We consider a system (3.1) with $C^{k, \mu}$ coefficients on $[0, T]$. We assume that the system is weakly hyperbolic and uniformly diagonalizable.

\subsection{Proof of Theorem 1.4}

The multiplicity of the eigenvalues is at most $N$ and we first prove Theorem 1.4 with $m=N$, that is with no assumption on the multiplicities.

Proposition 7.1. There is a family of approximate symmetrizers $S(t, \xi)$ which satisfy the properties (S1), (S2) and (S3) listed before Theorem 3.1 with parameter $\alpha=N(N-1) /(k+\mu+N(N-1))$.

Proof. We can assume that $|\xi| \geq 1$. For $\omega \in S^{d-1}$, denote by $S_{\varepsilon}(t, \omega)$ the approximate symmetrizers constructed in the previous section associated to the matrix $A(t, \omega)$. Then

$$
\begin{aligned}
& C^{-1} \Delta_{\varepsilon}(t, \omega) \operatorname{Id} \leq S_{\varepsilon}(t, \omega) \leq C \Delta_{\varepsilon}(t, \omega) \mathrm{Id}, \\
& \operatorname{Im}\left(S_{\varepsilon}(t, \omega) A(t, \omega) \leq C \varepsilon S_{\varepsilon}(t, \omega) .\right.
\end{aligned}
$$

Moreover, by (6.18),

$$
C^{-1} \varepsilon^{N(N-1)} \leq \Delta_{\varepsilon}(t, \omega) \leq C
$$

By Corollary 6.6, the coefficients $s_{\varepsilon, j, k}$ of $S_{\varepsilon}(t, \omega)$ are polynomials of the coefficients of $A$ and $A^{*}$, therefore are bounded in $C^{k, \mu}([0, T])$. By Proposition 4.4 they satisfy

$$
\left|\partial_{t} s_{\varepsilon, j, s}(t, \omega)\right| \leq \varphi_{j, k}(t, \varepsilon, \omega)\left|s_{\varepsilon, j, s}(t, \omega)\right|^{1-1 /(k+\mu)}
$$

with

$$
\left\|\varphi_{j, k}(\cdot, \varepsilon, \omega)\right\|_{L^{1}([0, T])} \leq C
$$

where $C$ is independent of of $\omega$ and $\varepsilon$. Using (7.1) and (7.3) we obtain that

$$
\left|\partial_{t} s_{\varepsilon, j, s}\right| \leq \varphi_{j, k} \Delta^{1-1 /(k+\mu)} \leq \varphi_{j, k} \varepsilon^{-N(N-1) /(k+\mu)} \Delta .
$$

Thus, with $\varphi=\sum \varphi_{j, k}$,

$$
\begin{aligned}
\left|\left(\partial_{t} S_{\varepsilon}(t, \omega) u, u\right)\right| & \leq C \varphi \varepsilon^{-N(N-1) /(k+\mu)} \Delta|u|^{2} \\
& \leq \varphi(t, \varepsilon, \omega) \varepsilon^{-N(N-1) /(k+\mu)}\left(S_{\varepsilon}(t, \omega) u, u\right) .
\end{aligned}
$$


To finish the proof, we define

$$
S(t, \xi)=S_{\varepsilon}(t, \omega), \quad \omega=\frac{\xi}{|\xi|}, \quad \varepsilon=|\xi|^{-k /(k+\mu+N(N-1))}
$$

where the exponent is chosen to balance the term $\varepsilon^{-N(N-1) /(k+\mu)}$ in (7.5) and the term $\varepsilon|\xi|$ coming from the term $\operatorname{Im} S_{\varepsilon}(t, \omega) A(t, \xi)$. Their final contribution is $O\left(|\xi|^{(N(N-1) /(k+\mu+N(N-1))}\right)$.

With Theorem 3.1 this proposition implies Theorem 1.4 with $m=N$.

When the maximal multiplicity $m$ is less than $N$, we argue as in the proof of Theorem 5.4. We can restrict ourselves to $|\xi| \geq 1$ and it is sufficient to construct symmetrizers in a conical neighborhood of an arbitrary given point $\left(t_{0}, \xi_{0}\right)$ with $\left|\xi_{0}\right|=1$. Given such a point, there is such a neighborhood on which one can perform a block reduction of $A$ and write

$$
\tilde{A}(t, \xi):=P(t, \xi) A(t, \xi) P^{-1}(t, \xi)=\operatorname{diag}\left(A_{1}(t, \xi), \ldots, A_{m}(t, \xi)\right)
$$

where the diagonal blocks $A_{j}$ have a size $N_{j} \leq m$.

Each block $A_{j}$ has approximate symmetrizers given by Proposition 7.1. They satisfy

$$
\left\{\begin{array}{l}
\partial_{t} S_{j}(t, \xi) \leq\langle\xi\rangle^{\alpha_{j}} \varphi_{j}(t, \xi) S_{j}(t, \xi), \quad\left\|\varphi_{j}(\cdot, \xi)\right\|_{L^{1}} \leq C \\
\operatorname{Im} S_{j}(t, \xi) \tilde{A}_{j}(t, \xi) \leq C|\xi|^{\alpha_{j}} S_{j}(t, \xi) \\
C^{-1} \Delta_{j}(t, \xi) \operatorname{Id} \leq S_{j}(t, \xi) \leq C \Delta_{j}(t, \xi) \operatorname{Id}
\end{array}\right.
$$

where $\alpha_{j}=N_{j}\left(N_{j}-1\right) /\left(k+\mu+N_{j}\left(N_{j}-1\right)\right)$, and the $\Delta_{j}$ satisfy

$$
\left\{\begin{array}{l}
|\xi|^{-N_{j}\left(N_{j}-1\right)} \leq \Delta_{j}(t, \xi) \leq C, \\
\left|\partial_{t} \Delta_{j}(t, \xi)\right| \leq C \varphi_{j}(t)|\xi|^{\alpha_{j}} \Delta_{j}(t, \xi) .
\end{array}\right.
$$

Note that $\alpha_{j} \leq \alpha:=m(m-1) /(k+\mu+m(m-1))$.

Introduce

$$
\Delta(t, \xi)=\prod_{j=1}^{m} \Delta_{j}(t, \xi)
$$

and for $j \geq 1$

$$
\Delta_{j}(t, \xi)=\prod_{l \neq j} \Delta_{l}(t, \xi) .
$$

Consider the symmetrizer

$$
\tilde{S}(t, \xi)=\operatorname{diag}\left(\Delta_{1} S_{1}, \ldots, \Delta_{m} S_{m}\right) .
$$


Each block $\Delta_{j} S_{j}$ is $\approx \Delta \operatorname{Id}_{N_{j}}$, and thus there is $C$ such that

$$
C^{-1} \Delta(t, \xi) \operatorname{Id} \leq \tilde{S}(t, \xi) \leq C \Delta(t, \xi) \operatorname{Id},
$$

while $\Delta$ satisfies

$$
|\xi|^{-M} \leq \Delta(t, \xi) \leq C, \quad M=\sum N_{j}\left(N_{j}-1\right) .
$$

By (7.8) and (7.9), one has

$$
\left|\partial_{t} \tilde{S}(t, \xi)\right| \leq C \varphi(t, \xi)|\xi|^{\alpha} \tilde{S}(t, \xi)
$$

with $\varphi$ uniformly bounded in $L^{1}$. The scalar factor $\Delta_{j}$ commutes to the block $A_{j}$ and therefore

$$
\operatorname{Im}(\tilde{S}(t, \xi) \tilde{A}(t, \xi)) \leq C|\xi|^{\alpha} \tilde{S}(t, \xi) .
$$

Hence $\tilde{S}$ satisfies the properties (S1) (S2) and (S3) for $\tilde{A}$. Finally,

$$
S(t, \xi)=P^{*}(t, \xi) \tilde{S}(t, \xi) P(t, \xi)
$$

satisfies the properties (S1), (S3) and (S2). This finishes the proof of the construction of the symmetrizers and, with Theorem 3.1, the proof the Theorem 1.4 is complete.

\subsection{Remarks and additional results}

When $m=2$, the index $1+(k+\mu) / m(m-1)=1+(k+\mu) / 2$ is not optimal as shown in Theorem 1.6. Indeed, for $m=2$, we were able to take into account a more precise form of the symmetrizer to improve the estimate (7.5) of $\partial_{t} S$.

Moreover, Theorem 1.4 is interesting only for $k+\mu>m(m-1)$, since the general Bronštein index for uniformly diagonalizable system is 2 . This can be seen directly from the properties (6.3), (6.4) and (6.5) which imply that $S(t, \xi)=\Sigma_{\varepsilon}(t, \omega)$ with $\varepsilon=|\xi|^{-\frac{1}{2}}$ satisfy the conditions (S1) (S2) and (S3) with parameter $\alpha=1 / 2$. Thus the estimate (7.5) for $\partial_{t} S$ brings an improvement only for $k+\mu>m(m-1)$.

To conclude, we give a class of $N \times N$ systems, for which one can get the optimal index $1+k+\mu$, thus extending the optimal result of the $2 \times 2$ case. 
Assumption 7.2. We consider a weakly hyperbolic $N \times N$ system (3.1) with matrices $A_{j} \in C^{k, \mu}([0, T])$. For $t \in[0, T]$ and $\omega \in S^{d-1}$, we denote by $\Delta(t, \omega)$ the discriminant of the characteristic polynomial of $A(t, \omega)$ and by $A_{b}(t, \omega)=A(t, \omega)-\frac{1}{N}(\operatorname{tr} A(t, \omega))$ Id the traceless part of $A(t, \omega)$. We assume that there is a positive constant $\delta$ such that for all $t$ and $\omega$

$$
|\Delta(t, \omega)| \geq \delta\left|A_{b}(t, \omega)\right|^{N(N-1)} .
$$

Note that the discriminant $\Delta$ depends only on the traceless part $A_{b}$. Note also that $\Delta$ is an homogeneous polynomial of degree $N(N-1)$ of the coefficients of $A_{b}$ so that the inequality (7.16) is homogeneous in $A_{b}$. Moreover, the set of hyperbolic traceless matrices $A_{b}$ such that $\left|A_{b}\right|=1$ and $\Delta \geq \delta$ is a compact set of strictly hyperbolic matrices. Thus, by homogeneity, the set of hyperbolic matrices such that

$$
|\Delta| \geq \delta\left|A_{b}\right|^{N(N-1)}
$$

is a set of uniformly diagonalizable matrices.

The condition (7.16) implies that $A(t)$ is either strictly hyperbolic when $\Delta(t)>0$, or has an eigenvalue of multiplicity $N$ at points where $\Delta=0$.

Conversely, if $A$ is in a set of uniformly diagonalizable matrices, one has

$$
\left|A_{b}\right|^{2} \approx \sum \lambda_{j}^{2}
$$

where the $\lambda_{j}$ are the eigenvalues of $A_{b}$, while

$$
\Delta=\prod_{j<l}\left(\lambda_{j}-\lambda_{l}\right)^{2}
$$

Thus, in a set of uniformly diagonalizable matrices, the assumption (7.16) is equivalent to

$$
\forall j, l, \quad\left(\lambda_{j}-\lambda_{l}\right)^{2} \geq \delta_{1} \sum \lambda_{j}^{2}
$$

for some positive constant $\delta_{1}$.

Remark 7.3. For $2 \times 2$ systems, the assumption is equivalent to uniform diagonalizabilty.

Theorem 7.4. Under Assumption 7.2 the Cauchy problem 3.1 is well posed in Gevrey spaces $G^{s}$ with $s<1+k+\mu$. 
Proof. We use the symmetrizers $S_{\varepsilon}(t, \omega)$ as in the proof of Proposition 7.1. We show how one can improve the estimate of $\partial_{t} S_{\varepsilon}$, using (7.16). Indeed, $S_{\varepsilon}$ is an homogeneous polynomial of degree $N(N-1)$ of $\varepsilon$ and the coefficients of $A_{b}$ and their complex conjugate, denoted by $a=\left\{a_{\alpha}\right\}$. Therefore the derivative of its coefficients $s_{j, k}$ are linear combinations of terms of the form

$$
\varepsilon^{n} \partial_{t} a_{\alpha} q_{N(N-1)-n}(a), \quad 0 \leq n<N(N-1),
$$

where $q_{N(N-1)-n}$ is an homogeneous polynomial of degree $N(N-1)-n-1$ of $a$. Next we use Proposition 4.4 for each coefficient $a_{\alpha}$ :

$$
\left|\partial_{t} a_{\alpha}\right| \leq \varphi_{\alpha}(t)|a|^{1-1 /(k+\mu)}
$$

and obtain the bound

$$
\left|\partial_{t} s_{j, k}\right| \leq \varepsilon^{n} \varphi(t)|a|^{N(N-1)-n-1 /(k+\mu)} \leq \varepsilon^{n} \varphi(t)\left|A_{b}\right|^{N(N-1)-n-1 /(k+\mu)},
$$

where the $L^{1}$ norm of $\varphi$ is uniformly controlled by the $C^{k, \mu}$ norm of the coefficients. Using (7.16) and the bound (7.3) we conclude that

$$
\left|\partial_{t} s_{j, k}\right| \leq \varphi \varepsilon^{-1 /(k+\mu)} \Delta
$$

and thus

$$
\left|\partial_{t} S_{\varepsilon}\right| \leq \varphi \varepsilon^{-1 /(k+\mu)} S_{\varepsilon}
$$

which is (5.9). As in Proposition 5.2 we choose $\varepsilon=|\xi|^{-(k+\mu) /(k+\mu+1)}$ and thus obtain a family of symmetrizers which satisfy (S1) (S2) and (S3) listed before Theorem 3.1 with parameter $\alpha=1 / k+\mu+1$.

We see that the difference with the proof of the general case is that we have improved the exponent of $\varepsilon$ in the estimate of $\partial_{t} S$. For this, we have used Proposition 4.4 not for the coefficients of $S$ themselves, but, using their polynomial structure, to the coefficients of $A_{b}$. But this procedure is successful only because we have assume a control of these coefficients by $\Delta$, to obtain an estimate of $\partial_{t} S$ by $S$.

\section{References}

[Br] M.D Bronštein, The Cauchy Problem for hyperbolic operators with characteristic of variable multiplicity, Trans. Moscow. Math. Soc., 1982, pp 87-103. 
[CDGS] F.Colombini, E.De Giorgi, S.Spagnolo, Sur les équations hyperboliques avec des coefficients qui ne dépendent que du temps, Ann. Scuola Norm. Sup. Pisa Cl. Sci. 6 (1979), pp 511-559.

[CJS] F.Colombini, E.Jannelli, S.Spagnolo, Well-posedness in the Gevrey classes of the Cauchy problem for a nonstrictly hyperbolic equation with coefficients depending on time, Ann. Scuola Norm. Sup. Pisa Cl. Sci., 10 (1983), pp 291-312.

[CoNi] F.Colombini, T.Nishitani, Two by two strongly hyperbolic systems and Gevrey classes, Ann. Univ. Ferrara Sez. VII (N.S.) 45 (1999), suppl., (2000), pp 79-108.

[CNR] F.Colombini, T.Nishitani, J.Rauch, Weakly Hyperbolic Systems by Symmetrization, preprint,

[CS] F.Colombini, S.Spagnolo, An example of a weakly hyperbolic Cauchy problem not well posed in $C^{\infty}$, Acta Math. 148 (1982), pp 243-253.

[IvPe] V.Ivrii, V.Petkov, Necessary Conditions for the Cauchy Problem for Nonstrictly Hyperbolic Equations to be Well-posed, Uspekhi Mat. Nauk 29 (1974), pp 3-70; Russian Math. Surveys 29 (1974) pp1-70.

[Ka1] K.Kajitani, The Cauchy problem for nonstrictly hyperbolic systems in Gevrey Classes, J.Math.Kyoto Univ 23(1983), pp 599616.

[Ka2] K.Kajitani, The Cauchy problem for uniformly diagonalizable hyperbolic systems in Gevrey classes, in Hyperbolic Equations and Related Topics (Kataka/Kyoto, 1984). Boston Academic Press, 1986, pp 101-123.

[Ka3] K.Kajitani, The Cauchy problem for nonlinear hyperbolic systems, Bull. Sci . Math. 110 (1986), pp 3-48.

[Me] G.Métivier, $L^{2}$ well posed Cauchy Problems and Symmetrizability, J. Ecole Polytechnique, 1 (2014), pp 39-70.

[Ta1] S.Tarama, Une note sur les Systèmes Hyperboliques Uniformément Diagonalisables, Mem. Fac. Engrg. Kyoto Univ., 56 (1994), pp 9-18. 
[Ta2] S.Tarama, On the Lemma of Colombini, Jannelli and Spagnolo, Memoirs Fac. Engineering Osaka City University, 41 (2000), pp 111-116. 